\title{
Racial Health Disparities in a Cohort of 5,135 Transgender Veterans
}

\author{
George R. Brown • Kenneth T. Jones
}

Received: 20 May 2014 / Revised: 20 June 2014 / Accepted: 30 June 2014 / Published online: 16 July 2014

(C) W. Montague Cobb-NMA Health Institute (outside the USA) 2014

\begin{abstract}
Objective There are no large-scale studies of racial health disparities in transgender patients. The Veterans Health Administration (VHA) is the largest health-care system in the United States and was an early adopter of electronic health records. We present new data on medical and mental health disparities in a large population of transgender veterans.

Method Using four ICD-9-CM codes, we identified a cohort of transgender veterans who presented for VHA care from1996-2013. A total of 5,135 transgender veterans were identified, 387 were non-Hispanic Black and 4,120 were nonHispanic White. These two groups were compared to determine if medical and/or mental health disparities existed.

Results Black transgender veterans had increased odds to be diagnosed with alcohol abuse (adjusted odds ratio $[\mathrm{AOR}]=$ $1.86,95 \%$ confidence interval $[\mathrm{CI}]=1.50-2.31, p<0.0001$ ), benign prostatic hyperplasia $(\mathrm{AOR}=1.36, \mathrm{CI}=1.01-1.84$, $p<0.05)$, congestive heart failure $(\mathrm{AOR}=1.51, \mathrm{CI}=1.04$ $2.19, p<0.05)$, HIV/AIDS (AOR $=6.77, \mathrm{CI}=4.60-9.97$, $p<0.0001)$, hypertension $(\mathrm{AOR}=1.71, \mathrm{CI}=1.34-2.17$, $p<0.0001)$, end-stage renal disease $(\mathrm{AOR}=3.34, \mathrm{CI}=1.65$ $6.93, p<0.001)$, serious mental illness $(\mathrm{AOR}=1.35, \mathrm{CI}=1.09$ $1.68, p<0.01)$, and tobacco use (AOR $=1.29, \mathrm{CI}=1.04-1.59$, $p<0.05)$. However, the odds of Black transgender veterans to be diagnosed with the following conditions were reduced:
\end{abstract}

Disclaimer: The views expressed in this manuscript are those of the authors and do not necessarily reflect those of the United States Government or any of its departments or agencies, or the views of the affiliated academic institution (East Tennessee State University).

G. R. Brown $\cdot$ K. T. Jones

Office of Health Equity, Veterans Health Administration,

Washington, D.C., WA, USA

G. R. Brown $(\bowtie)$

Department of Psychiatry, East Tennessee State University, Johnson

City, TN, USA

e-mail: george.brown@va.gov depression $(\mathrm{AOR}=0.73, \mathrm{CI}=0.58-0.93, p<0.01)$, hypercholesterolemia $(\mathrm{AOR}=0.71, \mathrm{CI}=0.57-0.89, p<0.01)$, and obesity $(\mathrm{AOR}=0.79, \mathrm{CI}=0.63-0.98, p<0.05)$. Black transgender veterans' odds of having a history of incarceration was nearly three times larger $(\mathrm{AOR}=2.91, \mathrm{CI}=1.84-4.62, p<0.0001)$ and their odds of experiencing homelessness was nearly two times larger (AOR $=1.85, \mathrm{CI}=1.49-2.31, p<0.0001)$ than White transgender veterans. The odds of Black transgender veterans to live in a rural area was $65 \%$ less than that of White transgender veterans $(\mathrm{AOR}=0.35, \mathrm{CI}=0.27-0.46, p<0.0001)$.

Conclusions This is the first study to examine a large cohort of transgender patients for the presence of racial disparities in psychiatric and medical health outcome disparities using retrospective medical chart data. Black transgender veterans were found to have a greater likelihood of social disadvantage and prevalence of several mental and medical conditions compared to White transgender veterans. Racial disparities occurred in a context of global health disparities in transgender veterans, as a group, compared to non-transgender veterans. These findings may have implications for policy, prevention strategies, and health-care delivery in VA and other health-care systems.

Keywords Race · Transgender · Veterans · Disparity · MSC Codes: 62,65

\section{Introduction}

People who experience a substantial, enduring, and disturbing sense of incongruity between their anatomical/birth sex (objective) and their "felt"/(subjective) sense of masculine or feminine identity may meet psychiatric diagnostic criteria described in the Diagnostic and Statistical Manual of Mental Disorder, fourth edition, text revision [1] as gender identity disorder (GID) or gender identity disorder "not otherwise specified" (GID NOS). With the publication of DSM-V in 
May 2013 [2], the diagnosis of gender identity disorder is changed to gender dysphoria (GD) in recognition of the fact that those with this condition do not suffer from a disordered identity, but rather from a constellation of symptoms that are both recognizable and treatable [2]. The umbrella term "transgender" has been used as a descriptor (not a diagnosis) for those who experience incongruity between birth sex and subjective sense of gender and who may or may not meet the diagnostic criteria for a disorder. The World Health Organization's International Classification of Diseases, Ninth Revision, Clinical Modification (ICD-9-CM) [3] lists four codes which encompass those transgender persons whose symptoms are severe enough to warrant a formal diagnosis. Many transgender persons seek psychiatric and/or medical treatments (for example hormonal "reassignment" using exogenous hormonal treatments to facilitate physical changes of the other sex); some also seek surgical treatments (sex reassignment surgery) to bring their bodies more closely in alignment with their felt genders). As noted in a report by the Institute of Medicine [4], transgender persons are known to experience less access to health care, discrimination in some health-care systems, and a variety of medical and psychiatric health disparities compared to those who do not identify as transgender. These include higher incidences of suicidality, depression, anxiety disorders, HIV disease, tobacco-related illness, alcohol, drug, and nicotine dependence $[5,6]$. Rarely do these studies have a large enough sample size to examine racial disparities; however, in the case of HIV disease, the Black transgender population in the USA appears to be disproportionately affected $[7,8]$. Social determinants of health play a major role in negative health outcomes in this population as well, with high rates of poverty, sexual and physical assaults, incarceration, and homelessness [4, 9]. The combined effects of these determinants have been shown to have a deleterious effect on the health of already marginalized populations [10-14].

Research about transgender persons and their health-care concerns or outcomes in the USA has been limited largely to sources outside of health-care systems, including Internetbased anonymous surveys [15], convenience samples [5], or small studies utilizing focus-group methodologies $[16,17]$. The majority of those who participate in such studies are White. Larger-scale studies of transgender health have been limited to western European countries with centralized gender clinics and a single-payer model of health insurance, enabling capture of national data [18-20]. Such studies include a very high percentage of White patients and little information on other racial or ethnic groups. The largest retrospective study published to date on disparities in veterans with a diagnosis of GID or GID NOS was that of Blosnich et al. [21]. Although no matched control group was utilized and the results were not analyzed by race or ethnicity, the findings suggested that the rate of suicide-related events among GID/GID NOS- diagnosed veterans was 20 times higher than that for the general Veterans Health Administration (VHA) population. Discrimination in the health-care setting has been reported in national surveys of transgender persons, $20 \%$ of whom are veterans, with the result being decreased access to care or avoidance of needed primary care services [15].

A description of the substantial clinical and educational efforts undertaken by VHA to address the health-care needs of transgender veterans is detailed elsewhere [22]. VHA Directives published in 2011 [23] and 2013 [24] instruct all VHA facilities to provide a range of transgender health-care services to intersex and transgender veterans. In the absence of systematic gender identity data collection for veterans or for those serving in the armed forces, it remains unknown how many veterans would self-identify as transgender and take advantage of this access to care. As part of a comprehensive research program undertaken by the Office of Health Equity (OHE) in VHA to assess health disparities among transgender veterans, we examined existing VHA data to determine if there were racial disparities in the prevalence of psychiatric and medical conditions in transgender veterans. We sought to determine if health and health outcome disparities exist in this population who generally have greater and more equal access to medical and mental health services than for many transgender persons in the community.

\section{Methods}

\section{Data Sources}

Encounter data used for this study came from multiple sources: Medical SAS Inpatient (fiscal year [FY] 19982013) and Outpatient dataset (FY 2000-2013), and nonVHA care files (FY 1996-2013). These files are extracted data that are prepared annually from a centralized database containing integrated care data. Demographic information, service characteristics, and enrollment data were supplemented with data from VHA's Corporate Data Warehouse (CDW), the Health Eligibility Center (HEC) enrollment files, and the Vital Status Files (VSF). The CDW is a relational database that contains data from various VHA clinical and administrative systems. The HEC enrollment files contain data on veteran's eligibility and enrollment status. The VSF contains mortality data from multiple sources including data on patients from the Social Security Administration. A spreadsheet maintained by the VHA Planning System Support Group (PSSG) was used to classify each patient's residential area as urban or rural. The study protocol was approved by the East Tennessee State University/Mountain Home VA Institutional Review Board. 


\section{Study Design}

Only non-Hispanic Black and non-Hispanic White transgender veterans were included for this study. Recording of race and ethnicity data is optional at the time of enrollment. As such, race was assigned as found in the patient's electronic health record (EHR). Transgender identity was established by one of the following ICD-9-CM codes [3] listed during an encounter in the EHR: 302.3 (transvestic fetishism, TF), 302.5x (transsexualism), 302.6 (GID NOS), or 302.85 (GID in adolescents or adults). The expansion of the definition of "transgender veteran" in this study to include TF is consistent with conceptualizations of transgender identity on a continuum that changes over the life course $[25,26]$, such that those who engage in cross-dressing activities but who do not meet the ICD-9-CM criteria for transsexualism, GID, or GID NOS often view themselves as transgender and may, or may not, utilize transgender health services at some point in their lives. Person and Ovesey [27] described the phenomenon known as "secondary transsexualism," wherein those men who initially meet the TF criteria earlier in life would later present with persistent gender dysphoria consistent with transsexualism. The inclusion of those with TF expanded the transgender veteran population by $6 \%$. We completed all analyses with, and without, the TF group.

\section{Measures}

\section{Demographics}

First, patients were assigned the gender as contained in the VSF. Current marital status was based on information in the EHR. Patients who had more than one entry and whose last entry was divorced, separated, or single were listed as previously married. A patient was determined to have religious or spiritual involvement if the EHR contained an entry for this field that was not missing or unknown. Those patients who had atheist listed were classified as not having any religious affiliation. The last recorded VHA enrollment priority group was assessed. Military service during the following eras was examined: World War II, Korean Conflict, Vietnam War, and Gulf War. Finally, a flag for deceased was created for patients who had a date of death listed.

\section{Social Determinants of Health}

Several structural issues known to be associated with health disparities were assessed [28-32]. Rural patients were those who had a zip code in a rural area as defined by VHA PSSG. An indicator variable was created for additional insurance besides VHA coverage. Veterans with a history of homelessness were those who had either an ICD-9-CM diagnosis of V60.0 (lack of housing) or clinical stop codes in their EHR that indicated receipt of services designated for homeless patients. Veterans with a history of incarceration were those who had clinical stop codes in their EHR indicating receipt of services for incarcerated, or justice-involved, veterans. While income and education level are not collected universally for enrollees, some veteran's priority groups are indicative of low income.

\section{Service-Related Characteristics}

Indicator variables were created to determine whether a patient had a service-connected disability, was a combat veteran, had been exposed to Agent Orange, was a Purple Heart recipient, or was an experienced military sexual trauma (MST).

\section{Mental Health and Medical Illnesses}

Cohort definitions using ICD-9-CM classifications of various mental health and medical illnesses are maintained by the VHA. Patients were classified as having the condition if the relevant diagnostic code was present in EHR at least once. The selection of medical and psychiatric diagnoses was informed by the literature or by hypotheses based on the authors' experience with this patient population. For example, given the high rates of alcohol and substance abuse in transgender populations [4], we included codes for liver disease.

\section{Data Analysis}

Chi-square statistics and $t$ tests were used to examine differences in Black and White transgender veterans. Odds ratios (ORs) were used to assess whether race is associated with social determinants, service-related characteristics, and various psychiatric and medical diagnoses. A $95 \%$ confidence interval (CI) is reported for each OR. Models were further adjusted to control for age, marital status, and religious or spiritual affiliation. For these models, marital status and religious affiliation were entered as dichotomous variables while age was entered as a continuous variable. A dummy variable was created for wartime period served, where Vietnam era or earlier service was coded 1. Variance inflation factors and tolerance diagnostics were conducted to assess potential multicollinearity [33]. Only the main effect of race is reported and discussed in unadjusted and adjusted models. All data were analyzed using SAS Enterprise Guide 5.1 (Cary, NC).

\section{Results}

A total of 5,135 unique patients meeting the diagnostic criteria for inclusion in the veteran transgender cohort were identified, 
of which $7.5 \%$ were Black and $80.2 \%$ were non-Hispanic White. Race could not be determined for $4.3 \%$ of transgender veterans; therefore, these veterans were ineligible for the study analyses. The final analysis was limited to the Black and White transgender veterans $(N=4507)$. As noted in Table 1, the groups differed significantly in the distribution of age, religious/spiritual affiliation, marital status, and wartime era in which they served. The average age of White transgender veterans was 56.7 years $(\mathrm{SD}=13.2)$ compared to 51.2 years $(\mathrm{SD}=13.9)$ for Black transgender veterans $(p<0.0001)$. Black transgender veterans were more likely to be single/never married (46 vs. $25 \%, p<0.0001$ ) and more likely to report a religious/spiritual affiliation (82 vs. $73 \%, p<0.0001)$. Between 8 and $9 \%$ of transgender veterans served during multiple wartime periods. Nearly three-quarters $(72 \%)$ of White transgender veterans compared to $63 \%$ of Black transgender veterans served in Vietnam or earlier periods $(p<0.0001)$.
Racial differences in social determinants of health, to the extent these can be quantified in VHA databases, were noted. The odds of Black transgender veterans to live in a rural environment were $66 \%$ less than the odds for White transgender veterans. $(20$ vs. $43 \%, \mathrm{OR}=0.34, \mathrm{CI}=0.26-0.44$, $p<0.0001)$. However, Black transgender veterans' odds of homelessness ( 47 vs. $29 \%, \mathrm{OR}=2.14, \mathrm{CI}=1.73-2.64$, $p<0.0001)$ and incarceration ( 7 vs. $2 \%, \mathrm{OR}=3.65, \mathrm{CI}=$ $2.33-5.71, p<0.0001)$ was at least twice that of White transgender veterans. These findings were consistent in adjusted models.

In unadjusted models, there were no significant differences by race for service-connected disability status, having served in a combat role, or receipt of a purple heart. However, Black transgender veterans had an odds of Agent Orange exposure that was $45 \%$ less than that of White transgender veterans ( 4 vs. $8 \%, \mathrm{OR}=0.55, \mathrm{CI}=0.33-0.90, p<0.05$ ). However, the odds of experiencing sexual trauma while on active
Table 1 Demographic characteristics of transgender veterans seeking VHA care, FY 2013 and earlier

\begin{tabular}{|c|c|c|c|c|c|}
\hline \multirow[t]{2}{*}{ Characteristic } & \multicolumn{2}{|c|}{ Black $(N=387)$} & \multicolumn{2}{|c|}{ White $(N=4,120)$} & \multirow[t]{2}{*}{$X^{2}$} \\
\hline & $N$ & $\%$ & $N$ & $\%$ & \\
\hline \multicolumn{6}{|l|}{ Gender } \\
\hline Female & 117 & 30.23 & 1,255 & 30.46 & 0.01 \\
\hline Male & 270 & 69.77 & 2,865 & 69.54 & \\
\hline Marital status & & & & & $79.75 \dagger$ \\
\hline Married & 75 & 19.38 & 954 & 23.16 & \\
\hline Widowed & 11 & 2.84 & 205 & 4.98 & \\
\hline Previously married & 125 & 32.30 & 1,929 & 46.82 & \\
\hline Single, never married & 176 & 45.48 & 1,022 & 24.81 & \\
\hline Unknown/missing & 0 & 0 & 10 & 0.24 & \\
\hline Religious or spiritual affiliation & 318 & 82.17 & 2,997 & 72.74 & $16.16 \dagger$ \\
\hline \multicolumn{6}{|l|}{ Enrollment priority group } \\
\hline 1 & 116 & 29.98 & 1,225 & 29.73 & $13.97 \#$ \\
\hline 2 & 20 & 5.17 & 268 & 6.50 & \\
\hline 3 & 37 & 9.56 & 429 & 10.41 & \\
\hline 4 & 48 & 12.40 & 332 & 8.06 & \\
\hline 5 & 132 & 34.11 & 1,347 & 32.69 & \\
\hline 6 & 6 & 1.55 & 86 & 2.09 & \\
\hline 7 & 6 & 1.55 & 76 & 1.84 & \\
\hline 8 & 21 & 5.43 & 330 & 8.01 & \\
\hline Not prioritized & 1 & 0.26 & 27 & 0.66 & \\
\hline Wartime period served & & & & & $16.47 * *$ \\
\hline World War II & 8 & 2.07 & 84 & 2.04 & \\
\hline Korean conflict & 12 & 3.10 & 210 & 5.10 & \\
\hline Vietnam era & 224 & 57.88 & 2,675 & 64.93 & \\
\hline Gulf War & 104 & 26.87 & 799 & 19.39 & \\
\hline Multiple & 35 & 9.04 & 319 & 7.74 & \\
\hline Missing & 4 & 1.03 & 33 & 0.80 & \\
\hline Age (mean, SD) & 51.21 & 13.94 & 56.87 & 12.18 & $t=-8.05 \dagger$ \\
\hline
\end{tabular}


duty service was 1.33 times higher for Black transgender veterans ( 19 vs. $15 \%, \mathrm{CI}=1.01-1.74, p<0.05)$. These findings were not observed in adjusted models.

Table 2 displays the proportion and odds for a series of medical and mental health issues in Black and White transgender veterans. In unadjusted analyses, Black transgender veterans had an increased odds of alcohol abuse (48 vs. $33 \%$, $\mathrm{OR}=1.99, \mathrm{CI}=1.61-2.45, p<0.0001)$, tobacco use (57 vs. $47 \%, \mathrm{OR}=1.36, \mathrm{CI}=1.10-1.67, p<0.01)$, HIV infection (13 vs. $2 \%, \mathrm{OR}=7.42, \mathrm{CI}=5.11-10.77, p<0.0001)$, end-stage renal disease ( 3 vs. $1 \%, \mathrm{OR}=2.78, \mathrm{CI}=1.38-5.61, p<0.01$ ), and serious mental illness ( 41 vs. $33 \%, \mathrm{OR}=1.45, \mathrm{CI}=1.17$ $1.80, p<0.001)$ than White transgender veterans. However, odds was reduced for Black transgender veterans as compared to their White counterparts for diagnoses of obesity (37 vs.
$42 \%, \mathrm{OR}=0.76, \mathrm{CI}=0.61-0.94, p<0.05)$, chronic obstruction pulmonary disease $(\mathrm{COPD} ; 19$ vs. $26 \%, \mathrm{OR}=0.67, \mathrm{CI}=0.51-$ $0.87, p<0.01$ ), hypercholesterolemia ( 47 vs. $62 \%, \mathrm{OR}=0.55$, $\mathrm{CI}=0.45-0.68, p<0.0001)$, and depression ( 71 vs. $76 \%$, OR $=0.78, \mathrm{CI}=0.62-0.99, p<0.05)$. These relationships were also observed in the adjusted models with the exception of COPD where race was no longer significant. A significant relationship was observed for congestive heart failure (CHF) and hypertension but only in the adjusted models. Odds of CHF (10 vs. $9 \%$, adjusted OR [AOR]= $1.51, \mathrm{CI}=1.04-2.19, p<0.05)$ and hypertension (61 vs. $59 \%, \mathrm{AOR}=1.71, \mathrm{CI}=1.34-2.17, p<0.0001)$ was higher for Black transgender veterans. Figure 1 provides a Forest plot with associated effect sizes associated with these outcomes.

Table 2 Adjusted odds of mental health and medical illnesses for transgender veterans seeking VHA care, FY 20113 and earlier

\begin{tabular}{|c|c|c|c|c|c|c|c|c|}
\hline \multirow[t]{2}{*}{ Medical concern } & \multicolumn{2}{|c|}{ Black $(N=387)$} & \multicolumn{2}{|c|}{ White $(N=4,120)$} & \multicolumn{2}{|c|}{ Unadjusted odds ratio } & \multicolumn{2}{|c|}{ Adjusted odds ratio } \\
\hline & $N$ & $\%$ & $N$ & $\%$ & OR & $95 \% \mathrm{CI}$ & OR & $95 \% \mathrm{CI}$ \\
\hline Acute myocardial infarction & 9 & 2.33 & 133 & 3.23 & 0.71 & $0.36-1.41$ & 0.88 & $0.44-1.75$ \\
\hline Affective disorder & 209 & 54.01 & 2,313 & 56.14 & 0.92 & $0.74-1.13$ & 0.84 & $0.69-1.05$ \\
\hline Alcohol abuse & 184 & 47.55 & 1,290 & 31.31 & $1.99 \dagger$ & $1.61-2.45$ & $1.86 \dagger$ & $1.50-2.31$ \\
\hline Benign prostatic hyperplasia & 63 & 16.28 & 676 & 16.41 & 0.99 & $0.75-1.31$ & $1.36^{*}$ & $1.01-1.84$ \\
\hline Breast cancer & 3 & 0.78 & 30 & 0.73 & 1.07 & $0.32-3.51$ & 1.10 & $0.33-3.65$ \\
\hline Cardiac arrest & 2 & 0.52 & 48 & 1.17 & 0.44 & $0.11-1.82$ & 0.55 & $0.13-2.27$ \\
\hline Cerebral vascular disease & 26 & 6.72 & 365 & 8.86 & 0.74 & $0.49-1.12$ & 0.97 & $0.63-1.48$ \\
\hline Chronic obstruction pulmonary disease & 74 & 19.12 & 1,081 & 26.24 & $0.67 * *$ & $0.51-0.87$ & 0.81 & $0.62-1.07$ \\
\hline Cirrhosis & 7 & 1.81 & 98 & 2.38 & 0.76 & $0.35-1.64$ & 0.75 & $0.35-1.65$ \\
\hline Congestive heart failure & 38 & 9.82 & 376 & 9.13 & 1.08 & $0.76-1.54$ & $1.51^{*}$ & $1.04-2.19$ \\
\hline Depression, major & 176 & 45.48 & 2,006 & 48.69 & 0.88 & $0.71-1.08$ & 0.85 & $0.69-1.05$ \\
\hline Depression, other & 275 & 71.06 & 3,124 & 75.83 & $0.78 *$ & $0.62-0.99$ & $0.73 * *$ & $0.58-0.93$ \\
\hline Diabetes & 101 & 26.10 & 1,125 & 27.31 & 0.94 & $0.74-1.19$ & 1.19 & $0.93-1.53$ \\
\hline Eating disorders & 12 & 3.10 & 110 & 2.67 & 1.17 & $0.64-2.14$ & 1.16 & $0.63-2.14$ \\
\hline HIV & 49 & 12.66 & 79 & 1.92 & $7.42 \dagger$ & $5.11-10.77$ & $6.77 \dagger$ & $4.60-9.97$ \\
\hline Hypercholesterolemia & 182 & 47.03 & 2,537 & 61.58 & $0.55 \dagger$ & $0.45-0.68$ & $0.71 * *$ & $0.57-0.89$ \\
\hline Hypertension & 237 & 61.24 & 2,439 & 59.20 & 1.09 & $0.88-1.35$ & $1.71 \dagger$ & $1.34-2.17$ \\
\hline Ischemic heart disease & 78 & 20.16 & 1,009 & 24.49 & $0.79 \#$ & $0.60-1.01$ & 1.08 & $0.82-1.44$ \\
\hline Obesity & 138 & 35.66 & 1,740 & 42.23 & $0.76^{*}$ & $0.61-0.94$ & $0.79 *$ & $0.63-0.98$ \\
\hline Panic disorder & 31 & 8.01 & 392 & 9.51 & 0.83 & $0.57-1.21$ & $0.71 \$$ & $0.49-0.97$ \\
\hline Posttraumatic stress disorder & 152 & 39.28 & 1,609 & 39.05 & 1.01 & $0.82-1.25$ & 0.94 & $0.75-1.16$ \\
\hline Prostate cancer & 16 & 4.13 & 156 & 3.79 & 1.10 & $0.65-1.85$ & 1.56 & $0.90-2.70$ \\
\hline Renal disease, chronic & 59 & 15.25 & 628 & 15.24 & 1.00 & $0.75-1.34$ & $1.29 \&$ & $0.96-1.75$ \\
\hline Renal disease, end stage & 10 & 2.58 & 39 & 0.95 & $2.78 * *$ & $1.38-5.61$ & $3.34 * * *$ & $1.65-6.93$ \\
\hline Serious mental illness & 160 & 41.34 & 1,347 & 32.69 & $1.45^{* * *}$ & $1.17-1.80$ & $1.35^{* *}$ & $1.09-1.68$ \\
\hline Suicidal ideation/attempt & 82 & 21.19 & 793 & 19.25 & 1.13 & $0.87-1.46$ & 0.97 & $0.75-1.26$ \\
\hline Tobacco use & 212 & 54.78 & 1,943 & 47.16 & $1.36^{* *}$ & $1.10-1.67$ & $1.29 *$ & $1.04-1.59$ \\
\hline Traumatic brain injury & 33 & 8.53 & 323 & 7.84 & 1.10 & $0.75-1.59$ & 0.96 & $0.65-1.40$ \\
\hline
\end{tabular}

Adjusted odds ratios control for age, marital status, religious or spiritual affiliation, and wartime period served

${ }^{*} p<0.05 ;{ }^{* *} p<0.01 ;{ }^{* * *} p<0.001 ; \dagger p<0.0001 ; \# p=0.07 ; \$ p=0.09 ; \& p=0.10$ 
Fig. 1 Forest plot of differences in lifetime mental health and medical illnesses for transgender veterans seeking VHA care, FY 2013 and earlier

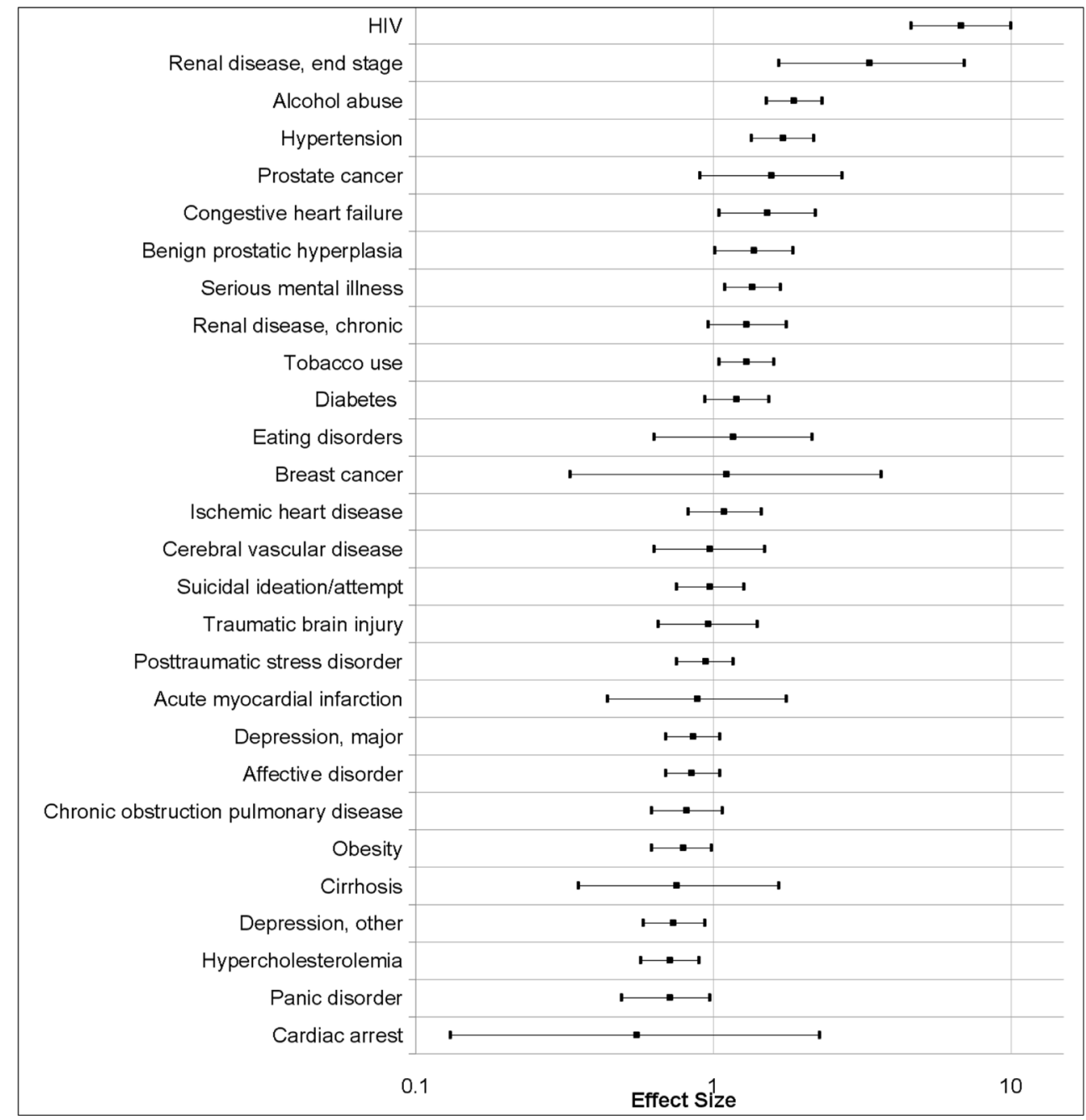

\section{Discussion}

This study is the first to examine racial disparities in health, health outcomes, and social determinants of health in a large cohort of transgender veterans. The results reported here generally support the conclusion that Black transgender veterans have similar or worse outcomes on a variety of measures of medical and mental health, as well as on some social determinants of health, than White transgender veterans. These disparities occur in a large integrated health-care system that provides access for veterans at more than 1,700 hospitals, clinics, community living centers, domiciliaries, and other facilities across the USA and its territories, based on broad eligibility requirements that exclude few veterans who have been honorably discharged from the Armed Forces of the USA. As noted by Saha [34], this feature makes the VA a good environment in which to study racial disparities without the usual confounds of insurance coverage or other measures of the ability to pay for care. Racial and ethnic minority veterans are often more dependent on VA as their primary source of health care than White veterans [35]. In spite of arguably equal access to care, racial disparities in health outcomes for Black veterans in many, but not all, domains have been reported [36]. For example, a meta-analysis of VA studies conducted prior to 2008 reported that 60 of 94 studies selected for analysis revealed racial disparities [37].

It has long been known that racial disparities in mental health care access and quality exist [38]. Racial and ethnic minorities in the community generally have less access to mental health-care services and are more likely than Whites to receive poorer quality care [38]. It is also the case that quality of care and disparities of care in a given system may not be directly related. In a community study where patients had similar access to care, no racial disparities in care were found, even though the overall level of care was suboptimal [39]. In another analysis of the entire transgender veteran cohort compared to matched veteran controls [40], we found global disparities for transgender veterans irrespective of race/ethnicity, suggesting that this group, as a whole, experiences issues with factors other than access in the VA system. 
The current study shows additional disparities for Black transgender veterans with the White comparator groups' baseline already reflecting substantial disparities when compared to veterans without a gender-related diagnosis.

Reduced access to mental health services by Blacks is also the result of delaying care or failing to seek mental health care, even when it is available [41]. Evidence-based treatments for depression and anxiety disorders are more likely to be received by White patients in the community than by Blacks [42]. The finding that Black transgender veterans are less likely to receive a diagnosis of depression, but more likely to receive diagnoses in the category of "serious mental illness" (e.g., schizophrenia, bipolar disorder, schizoaffective disorder), may represent an actual difference in the prevalence of these diagnoses or may reflect biases in diagnosing patterns for these conditions by mental health-care providers. Studies of racially discordant treatment dyads in mental health treatment programs, for example, suggest that Blacks may derive benefit from being treated by Black clinicians [43, 44]. Blacks have been traditionally underrepresented among the ranks of psychiatrists and psychologists in the community [45]. The same pertains to VA clinicians. In FY 2011, $64 \%$ of physicians and $86 \%$ of psychologists were White in VHA [46]. The percentage of Black physicians was $4.67 \%$ in FY 2011, $1.64 \%$ of VHA nurses were Black men, and $14.55 \%$ were Black women. Only $5 \%$ of physical therapists and psychologists in VHA were Black in FY 2011.

Alternatively, Black transgender patients may be less likely to present for care of depressive conditions, but serious mental illnesses, such as paranoid schizophrenia or the manic phase of bipolar disorder, often "force" clinical presentation due to the significant disruptions in the fabric of a family or community wrought by these conditions when untreated. Of note, there were no racial differences in suicidal behavior in this transgender cohort, but this finding occurs in the context of markedly increased suicidality in the transgender cohort as compared to non-transgender veterans [21]. This finding needs to be considered in the context that there are wellknown racial differences in suicidality, with White Americans more likely to commit suicide than Black Americans in most age groups studied nationally [47].

Obesity is a nationwide problem in the USA, with nearly $70 \%$ of adults in the overweight or obese categories [48]. Obesity prevalence is highest among Blacks, who have the highest age-adjusted rate of obesity nationally [49]. Associated with obesity is the elevated risk for cardiovascular disease and diabetes mellitus in Black Americans as well [50]. These national trends were not replicated in this study. In fact, we noted a trend for Whites to be obese compared to the Black transgender veterans ( $p=0.05$, Table 2$)$. COPD was found to be more likely in White transgender veterans in spite of a lower likelihood of tobacco use. Franssen and colleagues [51] noted the association between COPD and obesity, which may be independent of smoking history. They hypothesize that altered adipose tissue functions in the context of obesity may interact with systemic inflammation and COPD systems in a complex way that could account for this observation. It is also possible that Black transgender patients may receive differential care that could include less likelihood of being weighed at a visit irrespective of the presence of obesity or a more complete accounting for tobacco use than in White transgender veterans.

The presence of a large disparity in HIV prevalence in Black Americans compared to White Americans is well known [52] and is replicated in this study of transgender veterans. HIV prevalence among Black transgender veterans is nearly four times greater than rates in the hardest-hit areas in the world including Sub-Saharan Africa [53]. This disparity is complex and is likely determined by a multifactorial combination of social and sex partner networks, differential risk behaviors based on race of partner(s), past histories of childhood sexual abuse, and other factors [54]. Risk-related factors alone do not appear to account for this marked disparity, as even in the presence of lower rates of HIV-related risk behaviors, HIV prevalence rates may remain high among Black men who have sex with men, a term that has often included (MTF) transgender individuals $[55,56]$. Transgender status independent of race may also be associated with elevated rates of HIV infection [7]. Racial disparities in HIV infection in transgender persons were noted by Clements-Nolle et al. [7] where $63 \%$ of African-American MTF transgender adults were HIV positive compared to $17 \%$ of White transgender participants. Saha [34] reported that greater "cultural distance" between providers of HIV care and patients living with HIV was associated with disparities that appeared not to be based on race or ethnicity. However, non-White patients had lower levels of trust, adherence to antiretroviral therapies, and lower rates of viral suppression [57]. Additional research should examine neighborhood and community-level factors that may contribute to HIV and other disparities.

It is well established that Blacks are overrepresented in the American prison system, constituting $38 \%$ of inmates in state prisons in 2011-2012 [58]. The chances of ever being incarcerated for Black men in the USA are nearly $29 \%$, compared to less than $5 \%$ for White men [59]. Likewise, transgender people irrespective of race are overrepresented in American state prisons [60]. A study of 392 transgender adults with equal representation of Black and White MTF individuals found that $65 \%$ had reported a lifetime history of incarceration [7]. The increased likelihood of incarceration for Black transgender veterans is consistent with the theme of Blacks' overrepresentation in prisons, even against a backdrop of transgender people being overrepresented. This study does not directly address the reasons for this finding, but given that Black transgender veterans have higher rates of alcohol abuse and serious mental illnesses, it is possible that these 
characteristics could contribute to an increased probability of involvement with law enforcement and subsequent incarceration.

Discrimination by clinicians based on race is one possible contributing explanation for some of the findings in this study, including delaying or not seeking care, as has been shown elsewhere [61, 62]. This discrimination may take the form of unconscious bias [63], conscious bias (e.g., racial stereotyping), and/or statistical discrimination. Statistical discrimination is a form of decision making that occurs in medical settings that allows for individual clinician judgment about how to treat a given set of symptoms or lab abnormalities presented by a patient with one set of characteristics compared to how another patient may be treated with the same complaints/symptoms [64]. While the VA has an advanced system of clinical reminders and engages in numerous outreach efforts to engage veterans in care, there is still considerable clinician judgment in the provision of medical and psychiatric care.

Plant [65] argued that lack of positive experiences with "outgroup members," for example White clinicians with Black patients, creates negative expectancies and anxiety based on racial differences. Although our research does not address this issue, we hypothesize that the addition of transgender status to the already existing racial and/or cultural discordance between VA clinicians and Black transgender patients could contribute to the disparities noted in our results. Issues of belonging to multiple stigmatized or vulnerable populations, e.g., racial, sexual, and gender minority status, may be relevant as well. The minority stress model, originally applied to lesbian women [66] and later expanded to other sexual minorities [67], suggests that membership in sexual and gender minorities is associated with chronic stress, from both societal forces (enacted stigma) as well as internalization of stigma (felt or self-stigma). It remains a hypothesis as to whether minority stress may be amplified by multiple memberships in vulnerable groups in this study and whether that is expressed in disparate health outcomes. Jones et al. [68] have extended the minority stress model to sexual minorities, inclusive of MTF transgender individuals, with multiple memberships in vulnerable groups.

Additional research is warranted on the effects of experiencing multiple adverse mental, physical, and social conditions especially among Black transgender patients. The term syndemics has been introduced as one concept to describe two or more health or social conditions (e.g., HIV and unstable housing) that operate in a synergistic manner and contribute to a greater health burden for marginalized groups [10-12]. Stall et al. [13] examined the concept in a sample of urban gay men. Their research showed that a greater number of psychosocial problems (i.e., illicit drug use, depression, childhood sexual abuse, and partner violence) increased the likelihood that gay men would engage in risky sex. A better understanding of the social and health conditions of transgender individual, the set of conditions operating synergistically and resulting in greater health disparities, and the mechanisms that fuel these conditions (i.e., stigma) is warranted. The finding that modifiable health risk factors/behaviors differentially present in the Black transgender veteran group (higher alcohol and tobacco use, higher HIV disease prevalence) suggests the need for targeted interventions for transgender people of color, mobilization of community resources and community health worker programs, and HIV prevention strategies. Recently, resiliency factors such as optimism and education were shown to buffer the effects of syndemic conditions and should be explored in interventions with transgender individuals who face multiple health and social problems [14].

\section{Limitations}

This research is consistent with the IOM report's recommendations for research that advances the knowledge and understanding of LGBT health [4]. Several limitations should be noted. First, due to reliance on a diagnosis-based definition of transgender status, this study likely underestimates the transgender veteran population. Therefore, generalizability of the findings may be limited to those with clinically significant levels of gender dysphoria, likely a minority of veterans who identify as transgender and receive their health care in VHA facilities $[69,70]$. Although the analyses conducted in this paper were derived from the largest cohort of transgender patients ever studied to date in the USA, the number of Black patients was limited to 387 veterans. Generalizing these findings to other health-care systems may not be appropriate due to the fact that those in this study were all veterans who, theoretically, have greater access to a large integrated healthcare system than many in the community. Finally, this study does not fully take into account care that is provided outside the VA, which may or may not be reported to, and documented by, VA clinicians. There is some suggestion that White patients are more likely to obtain some or all of their health care from non-VA sources, which could contribute to disparities in outcomes [46].

\section{Future Directions}

The Office of Health Equity in VHA plans to explore options for additional analyses of the transgender veteran cohort to understand the potential sources of the identified disparities. These directions may include a study comparing service utilization, pharmacy benefits utilization, moderator analyses to determine the potential sources of health disparities, and prospective studies to determine whether national initiatives put 
into place since 2011 have closed the disparity gaps in healthcare outcomes for transgender veterans. Racial disparities in access and utilization of services will be examined in these studies as well.

Acknowledgments The authors acknowledge the support of the Office of Health Equity, Veterans Health Administration, Department of Veterans Affairs, for this work.

Conflict of Interest Dr. Jones declares he has no conflict of interest. Dr. Brown has received speaker honoraria from Janssen and Sunovion.

Informed Consent No subjects were contacted for this study; therefore, informed consent was not required by the East Tennessee State University/Mountain Home VAMC Institutional Review Board.

\section{References}

1. American Psychiatric Association. Diagnostic and statistical manual of mental disorders (4th ed., text rev.). Washington, DC: American Psychiatric Press; 2000.

2. American Psychiatric Association. Diagnostic and statistical manual of mental disorders. 5th ed. Arlington: American Psychiatric Publishing; 2013.

3. WHO. World Health Organisation international statistical classification of diseases and related health problems, 9th Revision (ICD-9CM). Geneva: WHO; 1978.

4. National Institutes of Health. The health of lesbian, gay, bisexual, and transgender people: building a foundation for better understanding. Washington, DC: National Academy of Sciences; 2011.

5. McDuffie E, Brown G. 70 US veterans with gender identity disturbances: a descriptive study. Int J Transgenderism. 2010;12:21-30.

6. Cole C, O'Boyle M, Emory L, Meyer W. Comorbidity of gender dysphoria and other major psychiatric diagnoses. Arch Sex Behav. 1997;26(1):13-26.

7. Clements-Nolle K, Marx R, Guzman BA, Katz M. HIV prevalence, risk behaviors, health care use, and mental health status of transgender persons: implications for public health intervention. Am J Public Health. 2001;91:915-21.

8. Cruz TM. Assessing access to care for transgender and gender nonconforming people: a consideration of diversity in combating discrimination. Soc Sci Med. 2014. doi:10.1016/j.socscimed.2014. 03.032 .

9. Xavier J, Bobbin M, Singer E. A needs assessment of transgendered people of color living in Washington, DC. Int J Transgenderism. 2005;8:31-47.

10. Singer M. AIDS and the health crisis of the urban poor: the perspective of critical medical anthropology. Soc Sci Med. 1994;39(7):931-48.

11. Singer M. A dose of drugs, a touch of violence, a case of AIDS: conceptualizing the SAVA syndemic. Free Inquiry. 1996;24(2): 99-110.

12. Singer M, Clair S. Syndemics and public health: reconceptualizing disease in bio-social context. Med Anthropol Q. 2003;17(4):423-41.

13. Stall RD, Mills TC, Williamson J, Hart T, Greenwood G, Paul J, et al. Association of co-occurring psychosocial health problems and increased vulnerability to HIV/AIDS among urban men who have sex with men. Am J Public Health. 2003;93(6):939-42.

14. O'Leary A, Jemmott JJ, Stevens R, Rutledge SE, Icard LE. Optimism and education buffer the effects of syndemic conditions on HIV status among African American men who have sex with men. AIDS Behav. 2014. doi:10.1007/s10461-014-0708-0.

15. Grant JM, Mottet LA, Tanis J, Herman JL, Harrison J, Keisling M. National Transgender Discrimination Survey report on health and health care. Washington, DC: National Center for Transgender Equality and the National Gay and Lesbian Task Force; 2010.

16. Shipherd JC, Mizock L, Maguen S, Green KE. Male-to-female transgender veterans and VA health care utilization. Int J Sex Health. 2012;24:78-87.

17. Kenagy G. Transgender health: findings from two needs assessment studies in Philadelphia. Health Soc Work. 2005;30(1):19-26.

18. Campo J, Nujman H, Merckelbach, Evers C. Psychiatric comorbidity of gender identity disorders: a survey among Dutch psychiatrists. Am J Psychiatry. 2003;160:1332-6.

19. Hepp U, Kraemer B, Schnyder U, Miller N, Delsignore A. Psychiatric comorbidity in gender identity disorder. J Psychosomatic Res. 2005;58: 259-61.

20. Gooren LJ, van Trotsenburg MA, Giltay EJ, van Diest PJ. Breast cancer development in transsexual subjects receiving cross-sex hormone treatment. J Sex Med. 2013;12:3129-34. doi:10.1111/jsm. 12319.

21. Blosnich JR, Brown GR, Shipherd JC, Kauth M, Piegari RI, Bossarte RM. Prevalence of gender identity disorder and suicide risk among transgender veterans utilizing Veterans Health Administration care. Am J Public Health. 2013;10:37-2.

22. Mattocks KM, Kauth MR, Sandfort T, Matza AR, Sullivan JC, Shipherd JC. Understanding health-care needs of sexual and gender minority veterans: how targeted research and policy can improve health. LGBT Health. 2013;1(1):30-7.

23. U.S. Veterans Affairs, Veterans Health Administration. VHA Directive 2011-026: providing health care for transgender and intersex veterans, 2013. www.va.gov/vhapublications. Accessed 15 Feb 2014.

24. U.S. Veterans Affairs, Veterans Health Administration. VHA Directive 2013-003: providing health care for transgender and intersex veterans, 2013. www.va.gov/vhapublications/ViewPublication. asp?pub_ID=2863. Accessed 15 Feb 2014.

25. Bockting W, Coleman E. Developmental stages of the transgender coming out process: toward an integrated identity. In: Ettner R, Monstrey S, Eyler A, editors. Principles of transgender medicine and surgery. New York: Haworth; 2007. p. 185-208.

26. Docter R. Transvestites and transsexuals: toward a theory of crossgender behavior. New York: Springer; 1988.

27. Person E, Ovesey L. The transsexual syndrome in males: II. Secondary transsexualism. Am J Psychotherapy. 1974;28:174-93.

28. Greenberg GA, Rosenheck RA. Mental health and other risk factors for jail incarceration among male veterans. Psychiatr Q. 2009;80:41-53.

29. Kilbourne AM, Switzer G, Hyman K, Crowley-Matoka M, Fine MJ. Advancing health disparities research within the health care system: a conceptual framework. Am J Public Health. 2006;96:2113-21.

30. Metraux S, Clegg L, Daigh JD, Culhane DP, Kane V. Risk factors for becoming homeless among a cohort of veterans who served in the era of the Iraq and Afghanistan conflicts. Am J Public Health. 2013;103(suppl):S255-61.

31. Tsai J, Desai MU, Cheng AW, Chang J. The effects of race and other socioeconomic factors on health services use among American military veterans. Psychiatr Q. 2013; 1-13.

32. Wallace AE, Weeks WB, Wang S, Lee A, Kazis LE. Rural and urban disparities in health-related quality of life among veterans with psychiatric disorders. Psychiatr Serv. 2006;57:851-6.

33. O'Brien RM. A caution regarding rules of thumb for variance inflation factors. Qual Quant. 2007;41:673-90.

34. Saha S, Sanders DS, Korthuis PT, Cohn JA, Sharp VL, Haidet P, et al. The role of cultural distance between patient and provider in 
explaining racial/ethnic disparities in HIV care. Patient Educ Couns. 2011;85(3):e278-84.

35. Washington DL, Villa V, Brown A, Damron-Rodriguez J, Harada N. Racial/ethnic variations in veterans' ambulatory care use. Am J Public Health. 2005;95(12):2231-7.

36. Trivedi AN, Grebla RC, Wright SM, Washington DL. Disparity persists for important clinical outcomes despite improved quality of care in the Veterans Affairs health system. Racial Health Aff. 2011;30:707-15.

37. Saha S, Freeman M, Toure J, Tippens KM, Weeks, Ibrahim S. Racial and ethnic disparities in the VA health care system: a systematic review. J Gen Int Med. 2008;23(5):654-71.

38. Department of Health and Human Services. Mental health: a report of the surgeon general. Rockville: Department of Health and Human Services; 1999.

39. Asch SM, Kerr EA, Keesey J, Adams JL, Setodji CM, Malik S, et al. Who is at greatest risk for receiving poor-quality health care? NEJM. 2006;354:1147-56.

40. Brown G, Jones K. Mental health and medical outcome disparities in 5135 transgender veterans receiving health care in the Veterans Health Administration: a case-control study. Proceedings of the 32nd annual meeting of the Gay and Lesbian Medical Association. September 11th, 2014.

41. Kessler RC, Nelson CB, McGonagle KA, Liu J, et al. Comorbidity of DSM-III-R major depressive disorder in the general population: Results from the U.S. National Comorbidity Survey. Brit J Psychiatry Suppl. 1996;30:17-30.

42. Wang PS, Berglund P, Kessler RC. Recent care of common mental disorders in the United States: prevalence and conformance with evidence-based recommendations. J Gen Int Med. 2000;15:284-92.

43. Rosenheck R, Fontana A, Cottrol C. Effect of clinician-veteran racial pairing in the treatment of post-traumatic stress disorder. Am J Psychiatry. 1995; 152:555-63.

44. Rosenheck R, Selbyi CL. Participation and outcome in a residential treatment and work therapy program for addictive disorders: the effects of race. Am J Psychiatry. 1998;155:1029-34.

45. Manderscheid RW, Berry JT. Mental health, United States, 2004, US Department of Health and Human Services. Substance abuse and Mental Health Services Administration. http://mentalhealth.samhsa. gov/publications/allpubs/SMA06-4195. Accessed 22 Feb 2014.

46. Workforce Management and Consulting Office. Veterans Health Administration workforce succession strategic plan and national plan supplemental report 2013. Washington, DC: Workforce Management and Consulting Office; 2013. http://vaww.va.gov/WMC/docs/2013 VHA_Workforce_Succession_Strategic_Plan_EBook.pdf. Accessed 23 Mar 2014.

47. Baca-Garcia E, Perez-Rodriguez M, Keyes K, Oquendo M, Hasin D, Grant B, et al. Suicidal ideation and suicide attempts in the United States: 1991-1992 and 2001-2002. Mol Psychiatry. 2010;15:250-9.

48. Ogden CL, Carroll MD, Kit BK, Flegal KM. Prevalence of obesity in the United States, 2009-2010. NCHS Data Brief. 2012;82:1-8.

49. Flegal KM, Carroll MD, Ogden CL, Curtin LR. Prevalence and trends in obesity among US adults, 1999-2008. JAMA. 2010;303: 235-41.

50. Wilson PW, D'Agostino RB, Sullivan L, Parise H, Kannel WB. Overweight and obesity as determinants of cardiovascular risk: the Framingham experience. Arch Intern Med. 2002;162:1867-72.

51. Franssen FM, O'Donnell DE, Goossens GH, Blaak EE, Schols AM. Obesity and the lung: 5. Obesity and COPD. Thorax. 2008;63:1110-17.
52. Clerkin EM, Newcomb ME, Mustanski B. Unpacking the racial disparity in HIV rates: the effect of race on risky sexual behavior among Black young men who have sex with men (YMSM). J Behav Med. 2011;34:237-43.

53. Kaiser Family Foundation. 2013. http://kff.org/global-health-policy/ fact-sheet/the-global-hivaids-epidemic/. Accessed 12 Mar 2014.

54. Feldman MB. A critical literature review to identify possible causes of higher rates of HIV infection among young Black and Latino men who have sex with men. J Natl Med Assoc. 2010;102:1206-21.

55. Magnus M, Kuo I, Phillips G, Shelley K, Rawls A, Montanez L, et al. Elevated HIV prevalence despite lower rates of sexual risk behaviors among black men in the District of Columbia who have sex with men. AIDS Patient Care STDS. 2010;24: 615-22.

56. Maulsby C, Millett G, Lindsey K, Kelley R, Johnson K, Montoya D. HIV among black men who have sex with men (MSM) in the United States: a review of the literature. AIDS Behav. 2014;18(1):10-25.

57. Earl TR, Saha S, Lombe M, Korthuis PT, Sharp V, Cohn J, et al. Race, relationships, and trust in providers among black patients with HIV/AIDS. Soc Work Res. 2013;37(3):219-26.

58. Bureau of Justice Statistics. 2011. http://www.bjs.gov/content/pub/ press/p12acpr.cfm. Accessed 22 Apr 2014.

59. Harris CT, Steffensmeier D, Ulmer JT, Painter-Davis N. Are Blacks and Hispanics disproportionately incarcerated relative to their arrests? Racial and ethnic disproportionality between arrest and incarceration. Race Soc Probl. 2009;1:187-99.

60. Brown GR. Autocastration and autopenectomy as surgical selftreatment in incarcerated persons with gender identity disorder. Int J Transgenderism. 2010;12:31-9.

61. Balsa AI, McGuire TG. Prejudice, clinical uncertainty, and stereotyping as sources of health disparities. J Health Econ. 2003;22:89-116.

62. Van Ryn M, Burke J. The effect of patient race and socio-economic status on physicians' perceptions of patients. Soc Sci Med. 2000;50: 813-28.

63. Burgess DJ, Fu SS, van Ryn M. Why do providers contribute to disparities and what can be done about it? J Gen Int Med. 2004;19: 1154-9.

64. Smedley BD, Stith AY, Nelson AR. Unequal treatment: confronting racial and ethnic disparities in health care. Washington, DC: National Academies Press; 2003.

65. Plant EA, Devine PG. The antecedents and implications of interracial anxiety. Pers Soc Psychol B. 2003;29:790-801.

66. Brooks VR. The theory of minority stress. In: Brooks VR, editor. Minority stress and lesbian women. Lexington: Lexington Books; 1981. p. 71-90.

67. Meyer IH. Prejudice, social stress, and mental health in lesbian, gay, and bisexual populations: conceptual issues and research evidence. Psychol Bull. 2003;129:674-97.

68. Jones KT, Wilton L, Millett GA, Johnson WD. Formulating the stress and severity model of minority social stress for black men who have sex with men. In: McCree DH, Jones KT, O'Leary A, editors. African Americans and HIV/AIDS: understanding and addressing the epidemic. New York: Springer; 2010.

69. Harrison-Quintana J, Herman JL. Still serving in silence: transgender service members and veterans in the National Transgender Discrimination Survey. LGBTQ Policy J. 2012;13:1-13.

70. Gates G. How many people are lesbian, gay, bisexual and transgender? Los Angeles: Williams Institute; 2011. p. 1-8. 